Supporting Information

\title{
$\pi$-Conjugated Dendrimers Based on Bis(enediynyl)benzene Units
}

\author{
Gil Tae Hwang and Byeang Hyean Kim* \\ National Research Laboratory, Department of Chemistry, \\ Division of Molecular and Life Sciences, Pohang University of Science and Technology, \\ Pohang 790-784, Korea
}

Experimental procedures for the synthesis of dendrimers

Figure S1. ${ }^{1} \mathrm{H}$ NMR spectrum of $\mathbf{G 3}$ in DMSO- $d_{6}$ 


\section{Experimental procedures for the synthesis of dendrimers}

General. All commercially available chemicals were used without further purification; solvents were dried carefully and distilled prior to use. All reactions were performed using dry glassware under argon atmospheres. Analytical TLC was carried out on Merck $60 \mathrm{~F}_{254}$ silica gel plate and column chromatography was performed on Merck 60 silica gel (230-400 mesh). Melting points were determined on an Electrothermal IA 9000 series melting point apparatus and are uncorrected. Infrared (IR) spectra were recorded using a Bruker Vector 22 spectrometer. ${ }^{1} \mathrm{H}$ and ${ }^{13} \mathrm{C}$ NMR spectra were recorded using a Bruker Aspect $300 \mathrm{MHz}$ NMR spectrometer. FAB mass spectra were measured using a JEOL four-sector tandem mass spectrometer (JMS-HX/HX110A); MALDI-TOF mass spectra were measured using a PE Biosystems Voyager System 4095. Elemental analyses were measured by the Center for Integrated Molecular Systems (CIMS), POSTECH, Korea. Ultraviolet (UV) spectra were obtained using an HP 8452A diode array spectrometer using a 10-mm path quartz cell versus a pure-solvent reference. Fluorescence (FL) spectra were obtained using a PTI model D-104 microscope photometer.

General Procedure for Dibromoolefinations: The synthesis of 5 is representative. Dendrimer G2 (154 $\mathrm{mg}, 0.0928 \mathrm{mmol})$ was added to a solution of $\mathrm{CBr}_{4}(490 \mathrm{mg}, 1.48 \mathrm{mmol})$ and $\mathrm{PPh}_{3}(770 \mathrm{mg}, 2.94 \mathrm{mmol})$ in $\mathrm{CH}_{2} \mathrm{Cl}_{2}(9 \mathrm{~mL})$. After stirring at room temperature for $3 \mathrm{~h}$, the solution was extracted with $\mathrm{H}_{2} \mathrm{O}$ and dried. Column chromatography gave $\mathbf{5}(138 \mathrm{mg}, 51 \%)$ as a solid.

General Procedure for Sonogashira Reactions: The synthesis of G3 is representative.

Divergent Method: $\left(\mathrm{PPh}_{3}\right)_{4} \mathrm{Pd}(14.6 \mathrm{mg}, 0.0126 \mathrm{mmol})$ and $\mathrm{CuI}(2.4 \mathrm{mg}, 0.0126 \mathrm{mmol})$ were added to a solution of $5(122 \mathrm{mg}, 0.0420 \mathrm{mmol})$ and 1-ethynylbenzaldehyde $(3 ; 99.0 \mathrm{mg}, 0.756 \mathrm{mmol})$ in $\mathrm{Et}_{3} \mathrm{~N}(0.1 \mathrm{~mL})$ and DMF ( $4 \mathrm{~mL})$. Argon was bubbled through the mixture for $2 \mathrm{~min}$ and then the mixture was subjected ten times to a pump/purge cycle before being stirred at $45-50{ }^{\circ} \mathrm{C}$ for $3 \mathrm{~h}$. After evaporation of the solvents in vacuo, the residue was subjected to chromatography on a silica gel column to give $\mathbf{G 3}$ (55.9 mg, 36\%). Recrystallization from $\mathrm{CHCl}_{3}$ gave pure $\mathbf{G 3}$.

Convergent Method: $\left(\mathrm{PPh}_{3}\right)_{4} \mathrm{Pd}(49.0 \mathrm{mg} 0.0420 \mathrm{mmol})$ and $\mathrm{CuI}(8.1 \mathrm{mg}, 0.0425 \mathrm{mmol})$ were added to a solution of 5 (180 mg, $0.142 \mathrm{mmol})$ in DMF (6 mL) under nitrogen and then ethynyldibenzaldehyde 6 (655 $\mathrm{mg}, 1.70 \mathrm{mmol})$ and $\mathrm{Et}_{3} \mathrm{~N}(0.4 \mathrm{~mL})$ were added to the solution. After degassing, the reaction mixture was stirred at $45-50{ }^{\circ} \mathrm{C}$ for $2 \mathrm{~h}$ and monitored by TLC. Water was added to this solution and then the product was filtered off and purified by column chromatography. After evaporating the solvents, the product was dried in vacuo (152 mg, 29\%).

G1. Column chromatography $\left(\mathrm{SiO}_{2} ; \mathrm{CH}_{2} \mathrm{Cl}_{2}\right)$; yield: $76 \%$. M.p. $185-186{ }^{\circ} \mathrm{C}$ dec.; IR (film): 3041,2820 , 2201, 1683, 1535, 1449, 1399, 1251, 1165, 1121, 1087, 1023, 799, 750; ${ }^{1} \mathrm{H}$ NMR (300 MHz, DMSO- $d_{6}$ ): $\delta 10.00$ (s, 4H; CHO), 7.96 (s, 4H; ArH), 7.72 (d, $J=8.2 \mathrm{~Hz}, 8 \mathrm{H} ; \mathrm{ArH}), 7.55$ (d, $J=8.1 \mathrm{~Hz}, 8 \mathrm{H} ; \mathrm{ArH}), 7.24$ (s, 
$2 \mathrm{H} ; \mathrm{C}=\mathrm{CH}) ;{ }^{13} \mathrm{C}$ NMR $\left(75 \mathrm{MHz}\right.$, DMSO- $\left.d_{6}\right): \delta 191.4,191.2,144.2,136.5,135.9,135.7,132.2,132.1,129.6$, 129.6, 129.4, 128.8, 128.5, 103.4, 94.5, 92.4, 90.2, 88.9; FAB-MS (m/z): $643.3[\mathrm{M}+\mathrm{H}]^{+}$; Anal. Calcd for $\mathrm{C}_{46} \mathrm{H}_{26} \mathrm{O}_{4}: \mathrm{C}, 85.97 ; \mathrm{H}, 4.08$. Found: C, 85.61; H, 4.34.

4. Column chromatography $\left(\mathrm{SiO}_{2}\right.$; hexane/ $\left.\mathrm{CH}_{2} \mathrm{Cl}_{2}, 3: 1\right)$; yield: $53 \%$. M.p. $167-169^{\circ} \mathrm{C}$ dec.; IR (film): 3045 , 2219, 1603, 1496, 1398, 1279, 1205, 1163, 862, 822, 759; ${ }^{1} \mathrm{H}$ NMR (300 MHz, DMSO- $\left.d_{6}\right): \delta 7.94$ (s, 4H; $\mathrm{ArH}), 7.90-7.57$ (m, 16H; ArH), 7.26 and $7.24(2 \mathrm{~s}, 6 \mathrm{H} ; \mathrm{C}=\mathrm{CH}) ;{ }^{13} \mathrm{C}$ NMR (75 MHz, DMSO- $\left.d_{6}\right): \delta 142.5$, 136.1, 135.9, 135.6, 132.4, 131.5, 131.2, 130.8, 129.8, 128.4, 127.2, 126.5, 123.0, 103.9, 102.8, 101.9, 90.7, 90.5, 89.3, 88.8; FAB-MS $(\mathrm{m} / \mathrm{z}): 1258.3[\mathrm{M}+\mathrm{H}]^{+}$; found, 1257.6; Anal. Calcd for $\mathrm{C}_{50} \mathrm{H}_{26} \mathrm{Br}_{8}: \mathrm{C}, 47.44 ; \mathrm{H}$, 2.07. Found: $\mathrm{C}, 47.41 ; \mathrm{H}, 2.11$.

G2. Column chromatography $\left(\mathrm{SiO}_{2} ; \mathrm{CH}_{2} \mathrm{Cl}_{2} / \mathrm{EtOAc}, 10: 1\right)$; yields: $31 \%$ from 2 and $33 \%$ from 4. M.p. > $256{ }^{\circ} \mathrm{C}$ dec.; IR (film): 3046, 2825, 2192, 1708, 1645, 1501, 1387, 1270, 1013, 972, 809, 738; ${ }^{1} \mathrm{H}$ NMR (300 MHz, DMSO- $\left.d_{6}\right): \delta 10.02$ and 10.00 (2s, 8H; CHO), 8.05 (d, $\left.J=8.4 \mathrm{~Hz}, 4 \mathrm{H} ; \mathrm{ArH}\right), 7.97-7.75$ (m, 24H; ArH), 7.72-7.61 (m, 24H; ArH), 7.27-7.24 (m, 6H; C=CH); ${ }^{13} \mathrm{C}$ NMR (75 MHz, DMSO- $d_{6}$ ): $\delta$ 191.4, 191.3, 144.0, 143.5, 136.0, 135.7, 135.2, 135.1, 135.0, 132.6, 132.2, 132.0, 131.9, 131.8, 130.4, 129.7, 129.6, 129.1, 128.7, 128.6, 128.4, 127.9, 127.9, 127.8, 122.8, 104.5, 103.7, 103.1, 102.7, 102.9, 94.6, 93.2, 93.1, 92.3, 92.1, 89.8, 88.7, 88.0; FAB-MS $(m / z): 1659.1[\mathrm{M}+\mathrm{H}]^{+}$; Anal. Calcd for $\mathrm{C}_{122} \mathrm{H}_{66} \mathrm{O}_{8}: \mathrm{C}, 88.28 ; \mathrm{H}, 4.01$. Found: $\mathrm{C}, 87.89$; H, 4.29 .

5. Column chromatography $\left(\mathrm{SiO}_{2}\right.$; hexane/ $\left.\mathrm{CH}_{2} \mathrm{Cl}_{2}, 3: 1\right)$; yield: $51 \%$. M.p. $161-163{ }^{\circ} \mathrm{C}$ dec.; $\mathrm{IR}$ (film): 3057 , 2203, 1651, 1520, 1464, 1202, 1059, 850, 794, 744; ${ }^{1} \mathrm{H}$ NMR (300 MHz, DMSO- $\left.d_{6}\right): \delta 8.00$ (s, 4H; ArH), 7.88-7.62 (m, 48H; ArH), 7.27-7.24 (m, 14H; $\mathrm{C}=\mathrm{CH}) ;{ }^{13} \mathrm{C}$ NMR (75 MHz, DMSO- $d_{6}$ ): $\delta$ 144.0, 143.6, 136.2, 136.1, 136.1, 135.9, 135.2, 133.2, 132.9, 132.5, 131.7, 131.6, 131.4, 130.7, 130.6, 129.2, 129.1, 129.0, 128.5, 128.4, 128.1, 121.8, 121.7, 121.5, 104.1, 103.5, 103.4, 95.3, 95.1, 92.0, 91.7, 91.1, 90.5, 90.3, 90.1, 89.3, 88.1, 87.9, 84.8, 81.9; MALDI-TOF-MS $(\mathrm{m} / \mathrm{z})$ : [M] $]^{+}$calcd 2889.2; found, 2889.0.

G3. Column chromatography $\left(\mathrm{SiO}_{2} ; \mathrm{CH}_{2} \mathrm{Cl}_{2} / \mathrm{EtOAc}, 10: 1\right)$; yields: $29 \%$ from 4 and $36 \%$ from 5. M.p. > $267{ }^{\circ} \mathrm{C}$ dec.; IR (film): 3051, 2831, 2180, 1701, 1649, 1553, 1515, 1469, 1215, 833, 765, 701; ${ }^{1} \mathrm{H}$ NMR (300 MHz, DMSO- $d_{6}$ ): $\delta 10.02$ and 10.00 (2s, $\left.16 \mathrm{H} ; \mathrm{CHO}\right), 8.01$ (d, $\left.J=8.6 \mathrm{~Hz}, 4 \mathrm{H} ; \mathrm{ArH}\right), 7.96-7.83(\mathrm{~m}, 56 \mathrm{H}$; $\mathrm{ArH}$ ), 7.75-7.60 (m, 56H; ArH), 7.28-7.23 (m, 14H; $\mathrm{C}=\mathrm{CH}) ;{ }^{13} \mathrm{C}$ NMR (75 MHz, DMSO- $d_{6}$ ): $\delta$ 191.2, 191.1, 144.2, 144.1, 144.1, 143.6, 143.5, 136.3, 136.2, 136.1, 135.9, 135.2, 135.2, 135.1, 134.7, 134.5, 133.2, 133.1, 133.1, 133.0, 132.6, 132.6, 132.5, 132.4, 132.3, 132.2, 132.0, 131.9, 130.8, 130.3, 130.2, 130.1, 130.0, 129.9, $129.9,129.8,129.7,129.6,129.5,129.4,129.1,128.9,128.8,128.6,128.5,128.4,128.0,127.9,127.9,127.8$, 123.5, 123.3, 123.1, 122.8, 122.6, 104.9, 104.7, 104.1, 103.8, 103.2, 102.7, 102.5, 97.2, 95.6, 95.4, 93.1, 92.7, 92.5, 91.5, 91.4, 91.0, 90.7, 90.6, 90.3, 90.1, 89.0, 88.4, 88.0, 86.9, 84.0, 83.4; MALDI-TOF-MS (m/z): [M] $]^{+}$ calcd 3691.1; found, 3691.2; Anal. Calcd for $\mathrm{C}_{274} \mathrm{H}_{146} \mathrm{O}_{16}$ : C, 89.09; H, 3.98. Found: C, 88.67; H, 4.31. 


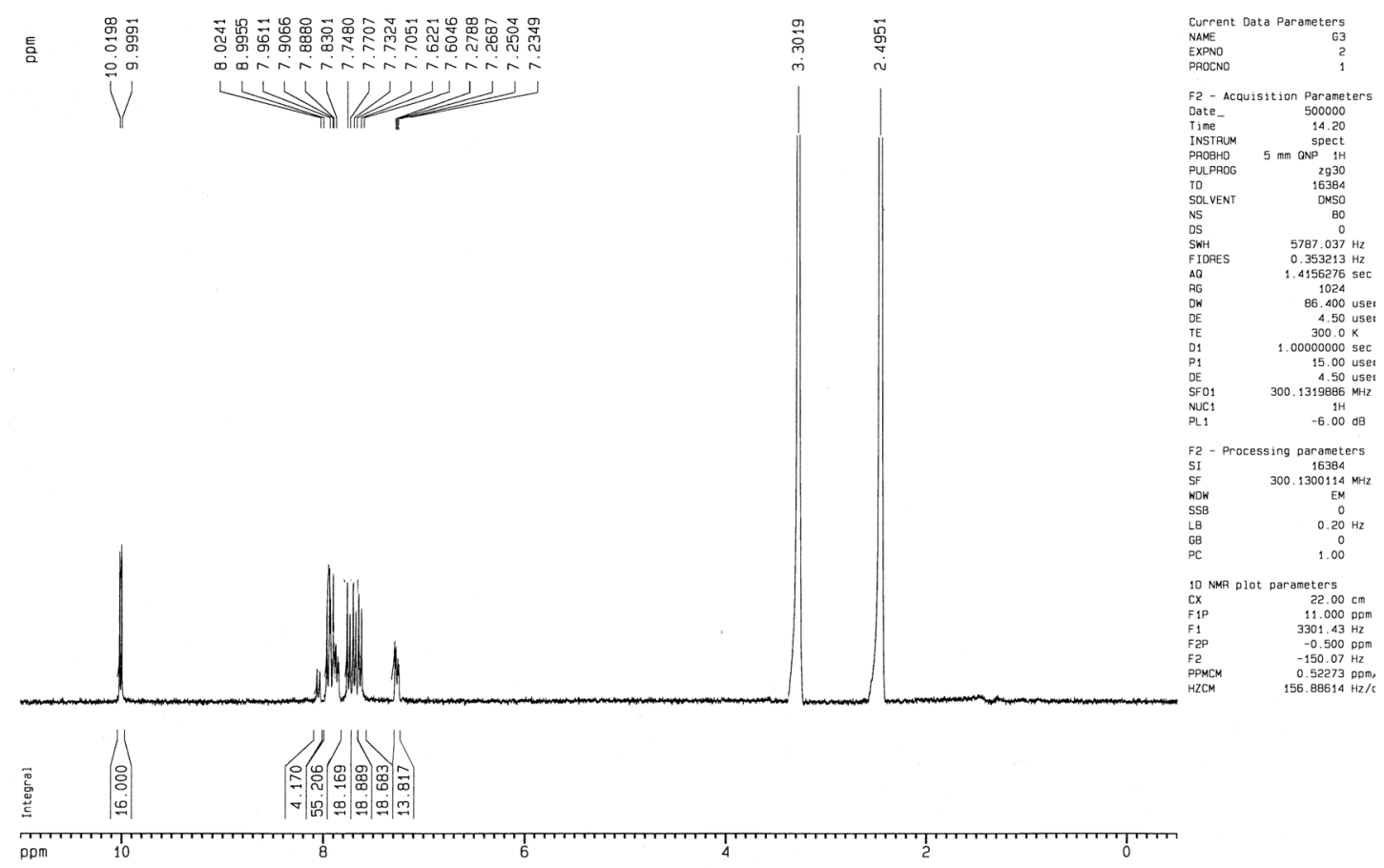

Figure S1. ${ }^{1} \mathrm{H}$ NMR spectrum of G3 in DMSO- $d_{6}$. 


\section{GPC analysis}

Column : PL mixed C*2 ,300*7.5 mm i.d., eluent : THF, flow rate : $0.8 \mathrm{~mL} / \mathrm{min}$, concentration : $2.0 \mathrm{mg} / \mathrm{mL}$ PS standards (g/mol) : 68 k, 30.9k, 22k, 11.5k, 7k, 3250, 690

Table S1. Molecular weights of G1-G3 obtained from GPC measurements

\begin{tabular}{lll}
\hline & $M_{\mathrm{n}}(\mathrm{g} / \mathrm{mol})$ & $M_{\mathrm{w}} / M_{\mathrm{n}}$ \\
\hline $\mathbf{G 1}$ & 540 & 1.07 \\
$\mathbf{G 2}$ & 860 & 1.05 \\
$\mathbf{G 3}$ & 1500 & 1.07
\end{tabular}

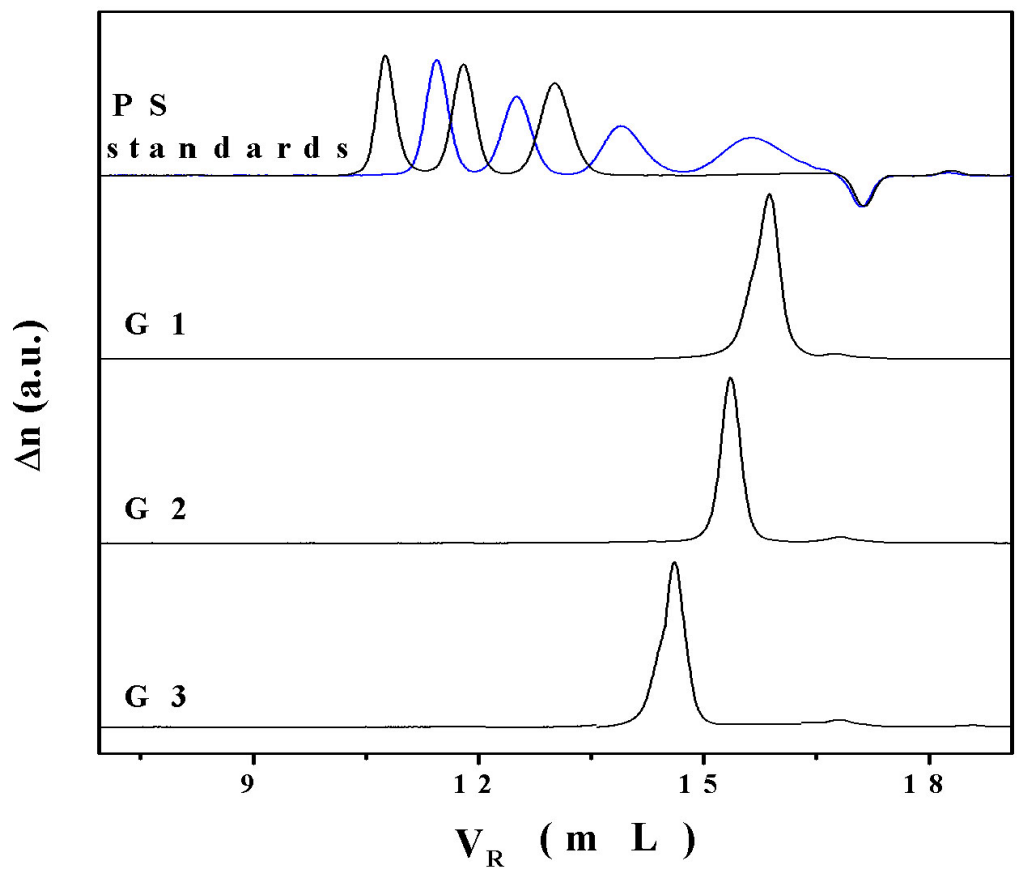

Figure S2. GPC traces of G1-G3. 